\title{
Estudio de viabilidad de un sistema para remoción de revoque para zonas productoras de pozos petroleros
}

\section{(Viability study of a system for filtercake remotion in pay zone of oil wells)}

\author{
Wang ZhaoZheng ${ }^{1}$, Fang $\mathrm{Xi}^{1}$, Henry Paúl Romero Cortez ${ }^{1}$, Fausto René Ramos \\ Aguirre $^{2}$
}

\begin{abstract}
Resumen:
En este artículo se exponen las ideas esenciales de un procedimiento experimental efectuado con el objetivo de comprobar si el sistema de remoción de revoque, es válido para ser usado en la zona productora. Este sistema se prepara en medio acuoso, y la concentración del removedor depende de la cantidad de revoque estimado en los pozos horizontales de petróleo para optimizar la limpieza de la zona productora y mejorar la producción de crudo. Los resultados de las pruebas realizadas demuestran que dicho sistema podría funcionar; pero se necesita evaluar la compatibilidad con los fluidos del pozo y con la roca en la zona productora.
\end{abstract}

Palabras clave: fluidos de perforación; removedor de revoque; pozos horizontales

\begin{abstract}
:
This article describes the essential ideas of an experimental procedure performed in order to check the filter-cake removal system is suitable for use like filter-cake removal system for pay zone. This system is made on aqueous phase and its concentration depend of the quantity of filtercake estimated in the wellbore of horizontal wells. This optimized the wellbore cleaning and increase the worth of well. The results of tests show that system could work, but it needs further analysis for determine the compatibility with fluids and rock from reservoir.
\end{abstract}

Keywords: drill-in; filter cake removal

\section{Introducción}

Durante la perforación de pozos petroleros se utilizan fluidos de perforación los cuales tienen diversas funciones como: controlar la presión de formación, remover los recortes del pozo, sellar las formaciones permeables encontradas durante la operación, enfriar y lubricar la broca, transmitir la energía hidráulica a las herramientas de fondo de pozo y a la broca, y quizás lo más importante, mantener la estabilidad y control del pozo (Al-Mehailani, 2009). Paralelamente a estas funciones el fluido forma un revoque en las paredes del pozo que luego necesita ser removido antes de iniciar la producción (Carrera, 2009), por tal motivo se diseña el sistema de remoción de revoque que en su esencia son sistemas ácidos que disuelven el carbonato de calcio y los polímeros presentes en el revoque.

Los antecedentes para la realización de este trabajo vienen dados por una cantidad de estudios y pruebas de laboratorio realizados por la empresa CNPC - Chuanquing Drilling Engineering Company Limited - CCDC Branch Ecuador para determinar si la formulación del sistema de

${ }^{1}$ CCDC Branch Ecuador, Quito - Ecuador ( \{wzhaozheng2004, fangxi, hromero\} @ccdc-ec.com )

2 Universidad Tecnológica Equinoccial, Quito - Ecuador (fausto.ramos@ute.edu.ec) 
remoción del revoque es válida. El removedor está basado en ácidos de acción débil para solubilizar el carbonato de calcio y los polímeros presentes en el revoque de los fluidos drill-in en un tiempo determinado (Leschi, 2006).

Las pruebas realizadas en esta investigación tienen el objetivo de sentar las bases del diseño de un sistema removedor de revoque (Mc Culloch, 2003), es decir encontrar experimentalmente la mejor formulación del sistema; de esta manera se pretende personalizar cada sistema para cada locación, campo y yacimiento, con el fin de obtener mejores rendimientos y maximizar la producción de los pozos y minimizar los daños en la formación (Davidson, 1997).

\section{Los objetivos específicos son:}

1) Identificar si el sistema puede utilizarse como removedor de revoque.

2) Identificar la concentración óptima del sistema.

3) Identificar las ventajas y desventajas del sistema.

\section{Metodología empleada}

Todas las pruebas de laboratorio fueron realizadas con los equipos y reactivos de la empresa CNPC - Chuanquing Drilling Engineering Company Limited - CCDC Branch Ecuador en las instalaciones de la UTE, en la ciudad de Quito.

Sistema de unidades:

- Masa: Libra (lb) y/o gramo (g)

- Volumen: Barril (bls) y/o mililitros (ml)

- Tiempo: minutos ( $\min )$

- $\mathrm{pH}$ : adimensional

- Temperatura: grados Celsius $\left({ }^{\circ} \mathrm{C}\right)$

- Porcentaje en peso: (\%p)

- Concentración: libras por barril (lb/bls) y/o gramos por mililitro (g/ml)

Principales pruebas efectuadas:

- Concentración de saturación en agua.

- Solubilidad máxima aproximada a condiciones atmosféricas de la ciudad de Quito en superficie del $\mathrm{CaCO}_{3}$ por cada $100 \mathrm{~g}$ de removedor.

- Variación del pH en función del tiempo y la temperatura.

- Remoción del revoque atmosférico.

Procedimientos experimentales empleados:

a) Concentración de saturación del sistema en agua.

- La prueba se realizó a condiciones atmosféricas de la ciudad de Quito, temperatura ambiente y presión atmosférica de la ciudad de Quito.

- Se colocó $10.00 \mathrm{~g}$ de removedor en $50 \mathrm{ml}$ de agua y agitó por $5 \mathrm{~min}$.

- Se observó si está disuelto el producto.

- Se colocó $1.00 \mathrm{~g}$ de removedor cada vez que se disuelve el producto por completo. 
- Se agitó por 5 minutos y se observó la disolución.

- Una vez que se observaron sólidos después de agitar se consideró que esa concentración está ligeramente pasada a la concentración de saturación.

- Se colocó $5 \mathrm{ml}$ de agua para disolver el exceso.

- Cuando se disolvió el removedor por completo esa fue otra concentración cercana a la saturación.

- Se promediaron los resultados.

b) Solubilidad del $\mathrm{CaCO} 3$ en el sistema.

- La prueba se realizó a condiciones atmosféricas de la ciudad de Quito, temperatura ambiente y presión atmosférica de la ciudad de Quito.

- Se utilizó $100.00 \mathrm{ml}$ de una solución del removedor $0.50 \mathrm{~g} / \mathrm{ml}$.

- Se mezcló $30.00 \mathrm{~g}$ de $\mathrm{CaCO} 3$ fino evitando derrames por la reacción.

- Se dejó reposar 8 horas para garantizar la máxima solubilidad del CaCO3.

- Se filtró el residuo y secó para medir la masa y se comparó con los $30.00 \mathrm{~g}$ iniciales.

c) Variación del pH en función del tiempo.

- La prueba se realizó en las siguientes condiciones: presión atmosférica de superficie y temperatura de fondo $80 \stackrel{\circ}{\circ}$.

- Se preparó diferentes soluciones con concentraciones de $2.00 \mathrm{lb} / \mathrm{bls}, 5.00 \mathrm{lb} / \mathrm{bls}, 10.00$ $\mathrm{lb} / \mathrm{bls}, 20.00 \mathrm{lb} / \mathrm{bls}, 30.00 \mathrm{lb} / \mathrm{bls}$ y solución saturada.

- Se midió el pH de las diferentes soluciones durante iguales intervalos de tiempo.

d) Remoción de revoque con diferentes concentraciones.

- La prueba se realizó en las siguientes condiciones: presión atmosférica de superficie y temperatura de fondo $80^{\circ} \mathrm{C}$.

- Se realizó la prueba de remoción de revoque atmosférico con las soluciones preparadas en el literal c.

- Se obtuvo el revoque de un fluido de perforación drill in utilizando el filtrado API según la norma API 13B.

- Se colocó el revoque dentro de la solución y observó la remoción.

- Se retiró el papel filtro restante y se secó para medir la masa y porcentaje de limpieza.

e) Remoción de los productos con diferentes concentraciones.

- La prueba se realizó en las siguientes condiciones: presión atmosférica de superficie y temperatura de fondo $80 \stackrel{\circ}{\circ}$.

- Se realizó la prueba de remoción de revoque atmosférico con las soluciones preparadas en el literal c.

- Se obtuvo el revoque de un fluido preparado con un solo producto utilizando el filtrado API.

- Se colocó el revoque dentro de la solución y observó la remoción.

- Se retiró el papel filtro restante y se secó para medir la masa y porcentaje de limpieza. 


\section{Resultados y Discusión}

Del estudio de solubilidad del sistema, puede deducirse que el removedor es soluble en agua hasta una concentración de $66.49 \mathrm{lb} / \mathrm{bls}$ aproximadamente; la solución obtenida es incolora y de olor leve. Los resultados de este método empleado son aproximados.

La Tabla 1 muestra los resultados de solubilidad del removedor en agua. Los valores en color rojo muestran las variaciones de su cristalización, valores que tomamos $(58.41 \mathrm{lb} / \mathrm{bls}, 75.10 \mathrm{lb} / \mathrm{bls}$, $65.97 \mathrm{lb} / \mathrm{bls}$ ) para determinar la solubilidad máxima promedio del sistema de remoción en agua y es de $66.49 \mathrm{lb} / \mathrm{bls}$.

Tabla 1. Solubilidad del removedor en agua

\begin{tabular}{|c|c|c|c|}
\hline $\begin{array}{c}\text { Removedor } \\
(\mathbf{g})\end{array}$ & Agua (ml) & Concentración (Ib/bls) & Residuo \\
\hline 10.00 & 50.00 & 70.09 & $\mathrm{Si}$ \\
\hline 10.00 & 60.00 & 58.41 & $\mathrm{Si}$ \\
\hline 10.00 & 70.00 & 50.06 & No \\
\hline 11.00 & 70.00 & 55.07 & No \\
\hline 12.00 & 70.00 & 60.08 & No \\
\hline 13.00 & 70.00 & 65.09 & No \\
\hline 14.00 & 70.00 & 70.09 & No \\
\hline 15.00 & 70.00 & 75.10 & No \\
\hline 16.00 & 70.00 & 80.11 & $\mathrm{Si}$ \\
\hline 16.00 & 71.00 & 78.98 & $\mathrm{Si}$ \\
\hline 16.00 & 72.00 & 77.88 & $\mathrm{Si}$ \\
\hline 16.00 & 73.00 & 76.82 & $\mathrm{Si}$ \\
\hline 16.00 & 74.00 & 75.77 & $\mathrm{Si}$ \\
\hline 16.00 & 75.00 & 74.77 & $\mathrm{Si}$ \\
\hline 16.00 & 80.00 & 70.09 & $\mathrm{Si}$ \\
\hline 16.00 & 85.00 & 65.97 & $\mathrm{Si}$ \\
\hline 16.00 & 90.00 & 62.31 & $\mathrm{No}$ \\
\hline
\end{tabular}

La Tabla 2 muestra los resultados de solubilidad del $\mathrm{CaCO} 3$ en una solución saturada del sistema de remoción. El estudio muestra que 45.06 gramos de $\mathrm{CaCO} 3$ se disuelven por cada $100.00 \mathrm{~g}$ de removedor.

Tabla 2. Solubilidad del $\mathrm{CaCO}_{3}$ en solución saturada del removedor

\begin{tabular}{|c|c|c|}
\hline Producto & Masa inicial (g) & Masa final (g) \\
\hline $\mathrm{CaCO}_{3}$ & 30.00 & 22.79 \\
\hline Sistema de remoción & 16.00 & \\
\hline $\mathrm{CaCO}_{3}$ disuelto & & 7.21 \\
\hline $\begin{array}{c}\text { Solubilidad } \\
\text { g de } \mathrm{CaCO}_{3} / 100 \text { g de removedor }\end{array}$ & & 45.06 \\
\hline
\end{tabular}

La Tabla 3 muestra los resultados de solubilidad aproximados de los productos utilizados en las dos formulaciones de fluidos en solución del sistema de remoción a condiciones atmosféricas de la ciudad de Quito; se realizaron pruebas de solubilidad para cada producto utilizado en la preparación del fluido de perforación (Nasr-El-Din, 2006). Con todos los productos se realizó el procedimiento del literal e.

Los estudios de la solubilidad de los polímeros en solución del sistema muestran que el PAC LV, PAC HV y XC HV son solubles mientras que los productos KPAN, NH4KPAN, SMP-1 y bentonita son insolubles. 
Tabla 3. Solubilidad de productos comerciales en el sistema de remoción de revoque.

\begin{tabular}{|c|c|c|c|c|c|c|}
\hline $\begin{array}{c}\text { Producto } \\
\text { (nombre } \\
\text { comercial) }\end{array}$ & $\begin{array}{c}\text { Tiempo } \\
(\mathbf{m i n})\end{array}$ & $\begin{array}{c}\text { Temperatura } \\
(\mathbf{(} \mathbf{C})\end{array}$ & $\mathbf{p H}$ & $\begin{array}{c}\text { Masa inicial } \\
(\mathbf{g})\end{array}$ & $\begin{array}{c}\text { Masa final } \\
(\mathbf{g})\end{array}$ & \%p de limpieza \\
\hline XC HV & 300 & 76.50 & 0.60 & 0.75 & 0.40 & 46.67 \\
\hline PAC LV & 300 & 75.40 & 0.57 & 0.74 & 0.07 & 90.54 \\
\hline PAC HV & 300 & 78.20 & 0.64 & 0.17 & 0.04 & 76.47 \\
\hline PAC LV /HV & 300 & 78.10 & 0.52 & 1.88 & 0.23 & 87.77 \\
\hline KPAN & 300 & 79.20 & 0.46 & 0.93 & 0.89 & 4.30 \\
\hline NH4KPAN & 300 & 75.30 & 0.45 & 0.99 & 0.95 & 4.04 \\
\hline SMP-1 & 300 & 76.90 & 0.54 & 1.23 & 1.22 & 0.81 \\
\hline Bentonita & 300 & 76.30 & 0.50 & 2.36 & 2.36 & 0.00 \\
\hline
\end{tabular}

\subsection{Variación del pH de una solución del removedor en función del tiempo.}

Se realizan dos formulaciones de fluidos de perforación la formulación 1, la cual contiene todos los productos utilizados por la empresa CNPC durante las operaciones de perforación y la formulación 2 contiene los productos utilizados en la perforación de la zona de interés o zona productora.

Se realiza la remoción del revoque obtenido con la formulación 1 con diferentes concentraciones del sistema de remoción. En la Figura 1 se muestran los valores de pH en función de la temperatura y tiempo. Los resultados del estudio de la variación del pH del sistema muestran que el $\mathrm{pH}$ se mantiene ácido desde el inicio y que no varía significativamente. Concentraciones menores a $2.00 \mathrm{lb} / \mathrm{bls}$ del sistema no tienen la cantidad suficiente de ácido para mantener la limpieza del revoque en el tiempo y el pH incrementa.

En la Tabla 4 mostramos los resultados de la remoción del revoque de la formulación 1 a diferentes concentraciones; se observa que en concentraciones menores a $10.00 \mathrm{lb} / \mathrm{bls}$ hay una limpieza parcial mientras que para las concentraciones superiores a $10.00 \mathrm{lb} / \mathrm{bls}$ se obtiene una limpieza más completa. En promedio se limpia el $47.38 \%$ p del revoque de la formulación 1 (todos los productos) con una concentración entre 10.00 y $30.00 \mathrm{lb} / \mathrm{bls}$ y un tiempo de limpieza de 5 horas.

Adicionalmente se realizan las pruebas de limpieza del revoque obtenido con el fluido de la formulación 2 utilizado en zonas productoras. Se observa en la figura 2 que el pH se mantiene ácido desde el inicio y no varía significativamente. El pH de la solución disminuye al aumentar la concentración del sistema. 


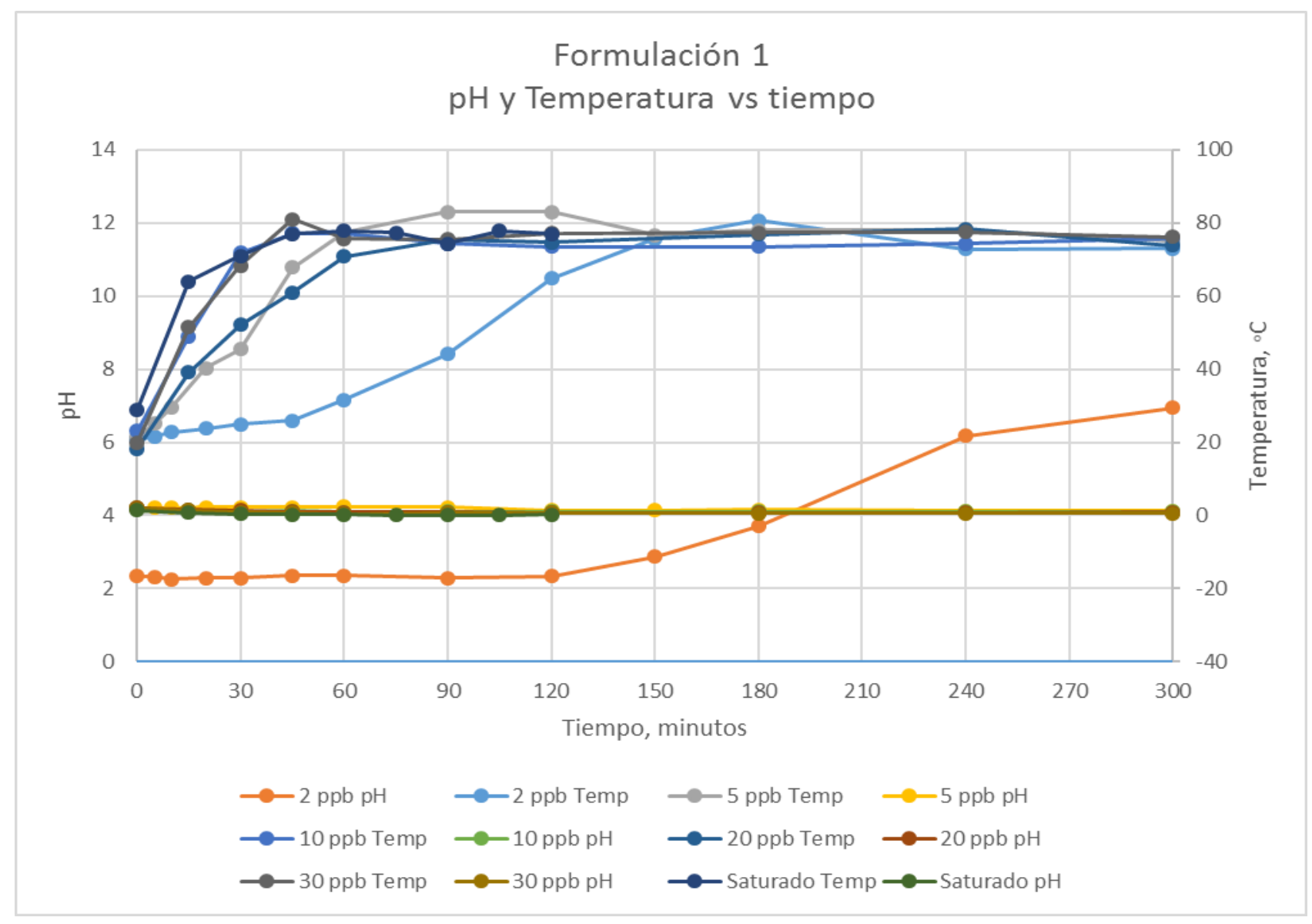

Figura 1. pH según temperatura y tiempo para formulación 1

Tabla 4. Remoción del revoque con Formulación 1

\begin{tabular}{|c|c|c|c|c|c|c|}
\hline $\begin{array}{c}\text { Concentración } \\
\text { del sistema } \\
(\mathbf{I b} / \mathbf{b} / \mathbf{s})\end{array}$ & $\begin{array}{c}\text { Tiempo } \\
(\mathbf{m i n})\end{array}$ & $\begin{array}{c}\text { Temperatura } \\
(\mathbf{(} \mathbf{C})\end{array}$ & $\mathbf{p H}$ & inicial & Final & Limpieza \\
\hline 2.00 & 300 & 80.70 & 2.25 & 1.97 & 1.74 & 11.68 \\
\hline 5.00 & 300 & 83.00 & 1.20 & 1.97 & 1.63 & 17.26 \\
\hline 10.00 & 300 & 75.80 & 0.92 & 1.97 & 1.08 & 45.18 \\
\hline 20.00 & 300 & 78.40 & 0.65 & 1.97 & 0.94 & 52.28 \\
\hline 30.00 & 300 & 81.00 & 0.51 & 1.97 & 1.09 & 44.67 \\
\hline 70.00 & 300 & 77.80 & 0.07 & 1.97 & 3.86 & ------ \\
\hline (saturado) & & & & & & \\
\hline
\end{tabular}




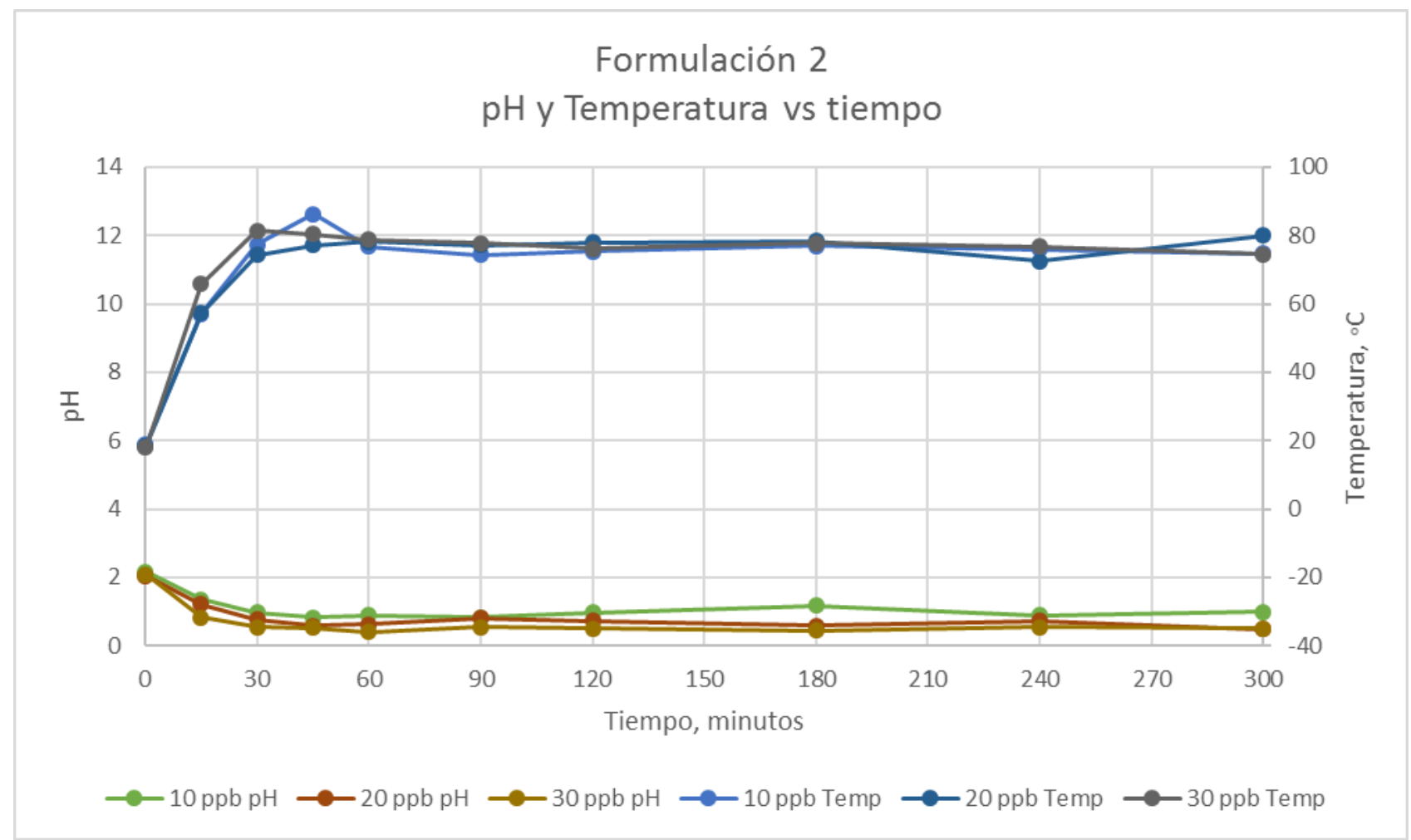

Figura 2. pH según temperatura y tiempo para formulación 2

Los revoques limpiados con concentraciones de 10.00, 20.00 y $30.00 \mathrm{lb} / \mathrm{bls}$ presentan una capa fina amarilla. En promedio se limpia el $93.23 \%$ p del revoque de la formulación 2 (productos utilizados en la zona productora) con una concentración entre 10.00 y $30.00 \mathrm{lb} / \mathrm{bls}$.

En la Tabla 5 se muestran los resultados del estudio de la variación del pH con el tiempo y la temperatura, además del porcentaje de limpieza obtenido para diferentes concentraciones del sistema de remoción. Podemos observar que el máximo porcentaje de limpieza se obtiene para la concentración de $30.00 \mathrm{lb} / \mathrm{bls}$.

Tabla 5. Remoción del revoque con Formulación 2

\begin{tabular}{|c|c|c|c|c|c|c|}
\hline \multirow{2}{*}{$\begin{array}{l}\text { Concentración } \\
\text { del sistema } \\
\text { (lb/bls) }\end{array}$} & \multirow{2}{*}{$\begin{array}{c}\text { Tiempo } \\
\text { (min) }\end{array}$} & \multirow{2}{*}{$\begin{array}{c}\text { Temperatura } \\
\left({ }^{\circ} \mathrm{C}\right)\end{array}$} & \multirow[b]{2}{*}{ pH } & \multicolumn{2}{|c|}{ Masa (g) } & \multirow{2}{*}{$\begin{array}{c}\text { Limpieza } \\
\% p\end{array}$} \\
\hline & & & & inicial & Final & \\
\hline 10.00 & 300 & 83.60 & 0.82 & 1.97 & 0.12 & 93.91 \\
\hline 20.00 & 300 & 79.90 & 0.48 & 1.97 & 0.18 & 90.86 \\
\hline 30.00 & 300 & 81.30 & 0.53 & 1.97 & 0.11 & 94.92 \\
\hline
\end{tabular}

En la Figura 3 mostramos la imagen de la limpieza del revoque que se obtiene utilizando el sistema de remoción de CNPC que ha sido objeto del estudio de este trabajo; esta imagen corresponde a la formulación 2 antes y después de haber actuado el sistema de remoción con concentración de $30.00 \mathrm{lb} / \mathrm{bls}$. Nótese cómo la limpieza obtenida es muy buena, por lo que los resultados de este trabajo son alentadores para seguir mejorando el sistema de remoción para su validación final. 


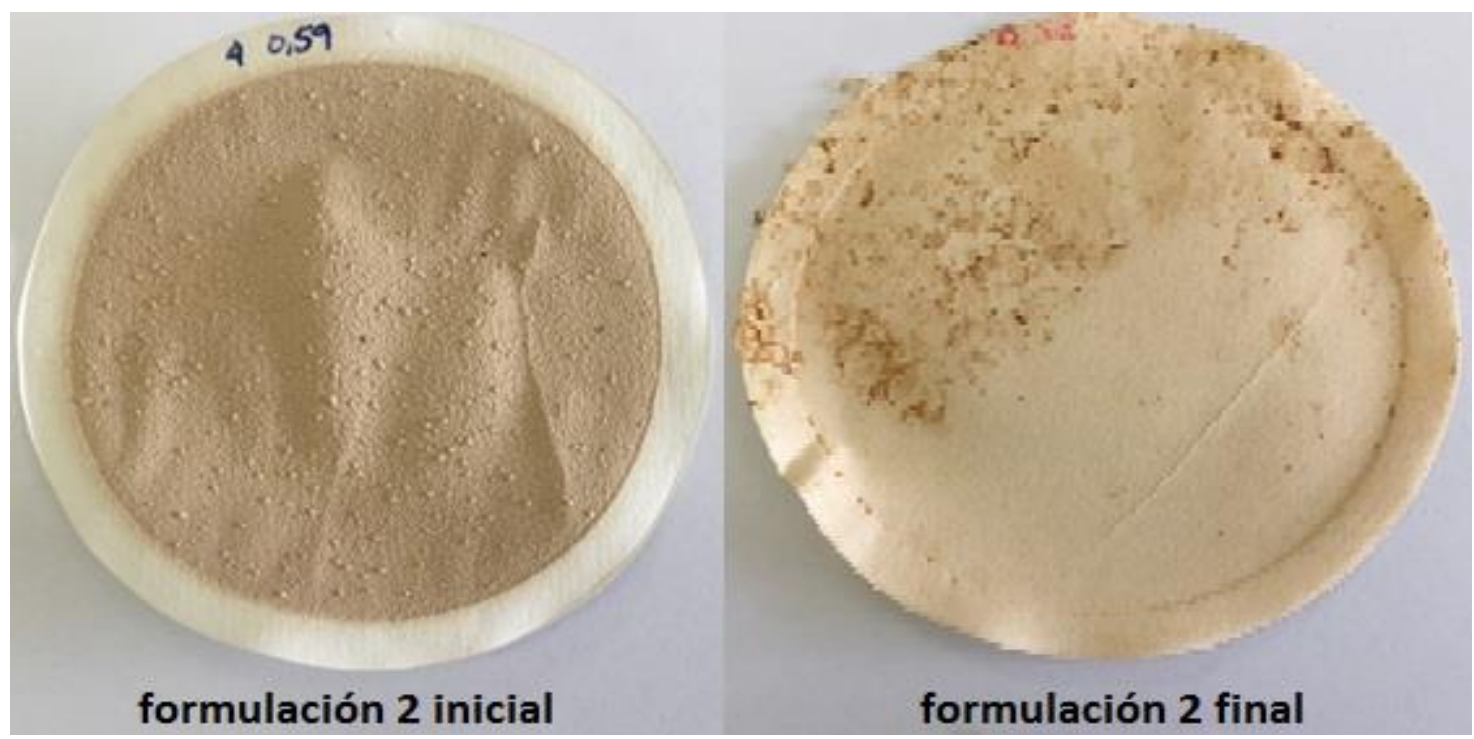

Figura 3. Limpieza del revoque de la formulación 2 utilizando el sistema de remoción de revoque de CNPC con concentración de $30.00 \mathrm{lb} / \mathrm{bls}$.

\section{Conclusiones}

El principio activo del sistema de remoción de revoque tiene una solubilidad aproximada en agua de $66.49 \mathrm{lb} / \mathrm{bls}$.

Para realizar una limpieza del revoque se necesita solubilizar el carbonato de calcio y los polímeros presentes; por esta razón es importante la solubilidad del carbonato de calcio en el sistema de remoción que es de $45.06 \mathrm{~g}$ de $\mathrm{CaCO} 3$ por cada $100.00 \mathrm{~g}$ de removedor.

Los polímeros PAC LV, PAC HV y XC HV son solubles en el sistema y los polímeros KPAN, NH4KPAN, SMP 1 y bentonita son insolubles en el sistema, esta información es útil para el diseño de la formulación 2 (formulación para la zona productora).

La formulación utilizada durante la perforación de los pozos es de suma importancia para el éxito de la remoción del revoque y podemos ver que la limpieza del revoque de la formulación 1 fue de $47.38 \%$ p mientras que la limpieza del revoque de la formulación 2 utilizada en la zona productora es del $93.23 \%$ p.

La concentración óptima del sistema de remoción de revoque es de 20.00 lb/bls.

\section{Recomendaciones}

El sistema de remoción de revoque de la empresa CNPC puede ser utilizado como rompedor de revoque en la zona productora, pero se debe utilizar un solvente mutual y un inhibidor de corrosión en la formulación.

Se deben realizar más pruebas de laboratorio como pruebas de break point utilizando el equipo de alta presión alta temperatura (HPHT) para identificar el tiempo de inicio de la remoción, pruebas de remoción de revoque atmosféricas con secuencia fotográfica, pruebas de remoción de revoque con una muestra real del fluido de perforación, pruebas de compatibilidad y un retorno de permeabilidad antes de hacer una prueba en campo.

Se recomienda utilizar el sistema como píldora liberadora de pega diferencial en fluidos con más de $40.00 \mathrm{lb} / \mathrm{bls}$ de carbonato de calcio con cualquier formulación. 


\section{Bibliografía}

Al-Mehailani, M., Al-Anzi, A., Gohain, A., Husain, I.,Haider, B., Davidson, E., MacMillan, B.(2009). Carefully Designed Water-Based Drill-In Fluid and Filter Cake Removal System Improve Reservoir Productivity and Reduce Well Completion Costs. Society of Petroleum Engineers, SPE 122327 1-8.

Carrera, C., Ferreira, C. (2009). Used the Time - Delayed Filter-Cake Removal System Significantly Improves Productivity Index in 20-Well Comparison. Society of Petroleum Engineers, SPE 122761. 1-9.

Davidson, E., Stewart S (1997). Open Hole Completions: Drilling Fluid Selection. Society of Petroleum Engineers SPE IADC 39284, 313-327.

Leschi, P., Demarthon, G., Davidson, E., Cnich,D (2006). Society of Petroleum Engineers, SPE 98164. Delayed Release Acid System for Cleanup of Al Khalij. Horizontal Open Hole Drains. Society of Petroleum Engineers SPE 98164, 1-9.

Mc Culloch, D.J., Mann, J., Mcmillan, P., Ali, S (2003). Damaged Removal in Screened Horizontal Wells. Society of Petroleum Engineers SPE 81732., 1-8.

Nasr-El-Din, H. A., Al-Otaibi, M.B., Al-Qahtani, A. A., Al -Fuwaires, A. A (2006). Filter-Cake Cleanup in MRC Wells using Enzyme Surfactant Solutions. Society of Petroleum Engineers SPE 98300.1-14. 